\title{
The Association between Nasal Septum Deviation and Sagittal Maxillary Growth Discrepancy
}

\author{
Ajay Bansal, Ashok Kumar Utreja, Satinder Pal Singh, Naresh Kumar Panda, Ashok Kumar Jena
}

\author{
ABSTRACT \\ Objective: To test the hypothesis that there is no association \\ between the nasal septum deviation and the sagittal growth \\ discrepancy of maxilla.
}

Materials and methods: Total 80 subjects in the age range of 7 to 51 years were divided into four groups of 20 each. Group I included subjects with normally positioned maxilla, group II included subjects with protruded maxilla, and group III included subjects with retruded maxilla in relation to the anterior cranial base. Subjects who already had a deviated nasal septum were included in group IV. All the subjects were clinically examined for the evaluation of nasal septum deviation. The deviation of nasal septum was categorized into anterior and posterior deviation. The sagittal maxillary growth discrepancy in all subjects was evaluated from lateral cephalograms. The association between the deviation of nasal septum and the discrepancy of maxillary growth in the sagittal plane was tested by Chi-square test.

Results: Deviated nasal septum was more common among subjects with normal and retruded maxilla than subjects with protruded maxilla. Subjects in whom nasal septum was already deviated, various sagittal maxillary growth discrepancies were equally distributed. There was no association between the nasal septum deviation and sagittal growth discrepancy of the maxilla $\left(\chi^{2}=2.143\right.$ and $\left.p=0.342\right)$.

Conclusion: There was no association between nasal septum deviation and the sagittal growth discrepancy of the maxilla.

Keywords: Nasal septum deviation, Sagittal maxillary growth discrepancy.

How to cite this article: Bansal A, Utreja AK, Singh SP, Panda NK, Jena AK. The Association between Nasal Septum Deviation and Sagittal Maxillary Growth Discrepancy. J Postgrad Med Edu Res 2012;46(2):98-101.

Source of support: Nil

Conflict of interest: None declared

\section{INTRODUCTION}

The nasal septum plays a significant role in the downward and forward displacement of maxilla. ${ }^{1-4}$ Scott reasoned that the nasal septum cartilage was more pressure tolerant tissue than sutures and had the capacity to push the whole nasomaxillary complex in downward and forward direction. ${ }^{1}$ However, Latham stated that the actual physical force for the maxillary displacement was at least in part, a pulling action of the septo-premaxillary ligament resulting from septal cartilage enlargement, rather than a pushing action. ${ }^{5}$ Enlow also emphasized that though the growth process is multifactorial, yet the nasal septum is still considered as the symbol for the force that causes displacement of nasomaxillary complex. ${ }^{6}$ On the other hand, Moss et al advocated that the nasal septum had only passive role in the displacement of maxilla. ${ }^{7,8}$ According to Moss et al, the forward and downward displacement of the maxilla is solely by the functional matrix mechanism without direct and purposeful participation by the expanding nasal septum. ${ }^{7,8}$ Thus, the role of nasal septum on the maxillary growth is controversial. If a positive association exists between the growth of nasal septum and the sagittal position of the maxilla, then the deviation of the nasal septum would also affect the normal sagittal position of the maxilla or an otherwise abnormal sagittal position of maxilla would result in nasal septum deviation. In the available literature, there are no uniform views on deviations of the nasal septum and their influence on the sagittal position of maxilla. ${ }^{9-11}$ Thus, the present study was designed to evaluate any association between the nasal septum deviation and the sagittal growth discrepancy of the maxilla.

\section{MATERIALS AND METHODS}

This cross-sectional study was conducted on $80(\mathrm{M}=45$, $\mathrm{F}=35$ ) North Indian subjects irrespective of their age and sex. Among these 80 subjects, 60 subjects $(M=28, F=32)$ were chosen from those who were undergoing treatment in the Unit of Orthodontics, Oral Health Sciences Centre, PGIMER, Chandigarh. These 60 subjects were selected based on the sagittal position of maxilla in relation to the anterior cranial base and were divided into three groups (Group I, II and III), each containing 20 subjects. The sagittal position of the maxilla was evaluated from SNA angle and $\mathrm{N}$ perpendicular to Point $\mathrm{A}(\mathrm{N} \perp \mathrm{A})$ linear distance. Group I (subjects in whom maxilla was positioned normally) included subjects who had SNA angle between $80^{\circ}-84^{\circ}$ and $\mathrm{N} \perp \mathrm{A}$ linear distance in the range of -2 to $-6 \mathrm{~mm}$. Those subjects who had SNA angle $>84^{\circ}$ and $\mathrm{N} \perp \mathrm{A}$ value $>-2 \mathrm{~mm}$ were included in group II (subjects in whom maxilla was protrusive). Subjects in whom the SNA angle was $<80^{\circ}$ and $\mathrm{N} \perp$ A linear distance was $<-6 \mathrm{~mm}$ were included in group III (subjects in whom maxilla was retrognathic). The remaining 20 subjects were chosen among the patients attending Otolaryngology OPD, PGIMER, Chandigarh, and these subjects were included in group IV. All the subjects of group IV had deviated nasal septum and were symptomatically severe enough to require surgical 
intervention. Lateral cephalograms were taken of all the subjects of group IV with teeth in occlusion and were used for the evaluation of sagittal position of maxilla. The deviation of nasal septum was evaluated clinically and the same otolaryngologist (NKP) evaluated all the subjects $(\mathrm{n}=80)$. Nasal septum deviations were classified as no deviation, anterior deviation and posterior deviation based on Cottle's functional areas of involvement ${ }^{12}$ (Fig. 1). Nasal septum was considered as normal or no deviation when there was equal space between the nasal septum and the inferior turbinate on both right and left side. The deviations (right or left) corresponding to the areas I, II and III and areas IV and $\mathrm{V}$ of the nasal septum as suggested by Cottle were considered as anterior and posterior deviations respectively. ${ }^{12}$ The study was conducted in accordance with the ethical guidelines of Helsinki declaration.

All the lateral cephalograms were recorded in the same machine (Kodak 8000C digital panoramic and cephalometric system). Same investigator (AB) traced all the lateral cephalograms. Only 10 cephalograms were analyzed on an average in a day to eliminate the error due to fatigue of investigator. The assessment of intraobserver variability and reproducibility of landmark location and measurement errors were analyzed by retracing $20 \%$ randomly selected lateral cephalograms after a gap of 21 days. The method error was calculated according to Dahlberg formula. ${ }^{13}$ A reliability of 0.93 was present for the reproducibility of landmarks location and measurements.

\section{STATISTICAL ANALYSIS}

The data was statistically analyzed by SPSS (version 15) software. The data was subjected to descriptive analysis.

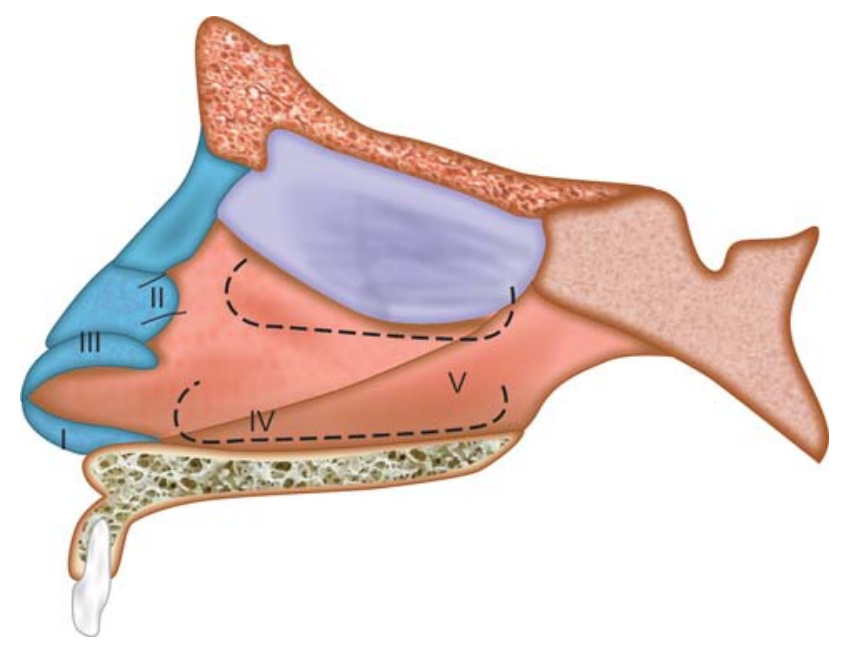

Fig. 1: Cottle's functional areas of the nose: (I) Anterior septal edge; (II and III) limen nasi; (IV) anterior edge of inferior turbinate; (V) posterior part of nose. Areas I, II and III are exclusively cartilaginous, and areas IV and $\mathrm{V}$ are consisting of both cartilage and bone including the vomeroethmoidal and the vomeromaxillary junction
Post-hoc test was used for multigroup comparisons. Chi-square test was performed to find out any association between independent variables, i.e. deviation of nasal septum and the discrepancy of sagittal maxillary growth. The p-value of 0.05 was considered as statistically significant.

\section{RESULTS}

Distribution of subjects in various groups according to their age and sex is shown in Table 1 . The distribution of subjects in groups I, II and III according to their type of nasal septum deviation is described in Table 2. Of all subjects of groups I, II and III (n = 60), 25 (41.67\%) subjects had no nasal septum deviation, whereas 23 (38.33\%) subjects had isolated anterior nasal septum deviation, three (5\%) subjects had isolated posterior nasal septum deviation and nine (15\%) subjects had both anterior as well as posterior deviation of nasal septum. Among all the subjects $(n=20)$ of group I, seven (35\%) subjects had no deviation of nasal septum, $10(50 \%)$ subjects had isolated anterior nasal septum deviation, one (5\%) subject had isolated posterior nasal septum deviation and two (10\%) subjects had both anterior as well as posterior deviation of nasal septum. Among all the subjects ( $n=20)$ of group II, $10(50 \%)$ subjects had no deviation of nasal septum, five (25\%) subjects had isolated anterior nasal septum deviation, two (10\%) subjects had isolated posterior nasal septum deviation and three (15\%) subjects had both anterior as well as posterior deviation of nasal septum. Among all the subjects $(n=20)$ of group III, eight (40\%) subjects had no deviation of nasal septum, eight (40\%) subjects had isolated anterior nasal septum deviation, four (20\%) subjects had both anterior as well as posterior deviation of nasal septum and none of the subjects had isolated posterior nasal septum deviation. Statistical analysis among subjects of groups I, II and III showed no association between discrepancy of maxillary growth in sagittal direction and the nasal septum deviation $\left(\chi^{2}=2.143\right.$ and $\mathrm{p}=0.342$ ). Among subjects of group IV in whom nasal septum was already deviated, 35\% each had normal and protruded maxilla, and 30\% subjects had retruded maxilla (Table 3).

\section{DISCUSSION}

The importance of the nasal septum in the development of midface has been mentioned in the literature with great frequency. ${ }^{1-5,14}$ The anatomical or acquired deformities of the nasal septum had a significant impact on the growth of midface; ${ }^{15}$ and most clinical studies had been conducted on children with a traumatic or congenital absence of the nasal septum ${ }^{5,7,16}$ and on young midfacial cleft patients with more or less severe dysjunction between the nasal septum and 


\begin{tabular}{|c|c|c|c|c|c|}
\hline \multirow[t]{2}{*}{ Groups } & \multirow[t]{2}{*}{ Subjects } & \multicolumn{2}{|c|}{ Sex distribution } & \multicolumn{2}{|c|}{ Age range in years } \\
\hline & & Male & Female & Minimum & Maximum \\
\hline 1 & 20 & 5 & 15 & 12 & 24 \\
\hline II & 20 & 9 & 11 & 10 & 28 \\
\hline III & 20 & 14 & 6 & 7 & 24 \\
\hline IV & 20 & 17 & 3 & 15 & 51 \\
\hline
\end{tabular}

\begin{tabular}{|c|c|c|c|c|c|}
\hline \multirow[t]{2}{*}{ Groups } & \multicolumn{4}{|c|}{ Distribution of subjects according to the type of nasal septum deviation } & \multirow{2}{*}{$\begin{array}{l}\text { Total } \\
n(\%)\end{array}$} \\
\hline & $\begin{array}{c}\text { No } \\
\text { deviation } \\
n(\%)\end{array}$ & $\begin{array}{c}\text { Anterior } \\
\text { deviation } \\
n(\%)\end{array}$ & $\begin{array}{c}\text { Posterior } \\
\text { deviation } \\
n(\%)\end{array}$ & $\begin{array}{c}\text { Both anterior and } \\
\text { posterior deviation } \\
n(\%)\end{array}$ & \\
\hline 1 & $7(35 \%)$ & $10(50 \%)$ & $1(5 \%)$ & $2(10 \%)$ & $20(100 \%)$ \\
\hline II & $10(50 \%)$ & $5(25 \%)$ & $2(10 \%)$ & $3(15 \%)$ & $20(100 \%)$ \\
\hline III & $8(40 \%)$ & $8(40 \%)$ & 0 & $4(20 \%)$ & $20(100 \%)$ \\
\hline
\end{tabular}

$\chi^{2}: 2.143 ;$ p: $0.342^{\text {NS}} ;$ NS: Nonsignificant

\begin{tabular}{cccc}
\multicolumn{4}{c}{ Table 3: Distribution of sagittal maxillary position among subjects $(\mathrm{n}=20)$ of group IV } \\
& Sagittal position of maxilla & Total $n(\%)$ \\
\cline { 1 - 2 } $\begin{array}{l}\text { Normal } \\
\mathrm{n}(\%)\end{array}$ & $\begin{array}{c}\text { Protruded } \\
\mathrm{n}(\%)\end{array}$ & $\begin{array}{c}\text { Retruded } \\
\mathrm{n}(\%)\end{array}$ & \\
\hline $7(35 \%)$ & $7(35 \%)$ & $6(30 \%)$ & $20(100 \%)$ \\
\hline
\end{tabular}

the maxillopalatal skeletal complex. ${ }^{17-19}$ As the nasal septum affects the growth and development of midface, ${ }^{20}$ so the deviation of nasal septum could also affect the growth of midface or deficiency of the maxillary growth could also affect the alignment of nasal septum.

In the present study although there was no statistically significant positive association between the discrepancy of maxillary growth in sagittal direction and the nasal septum deviation, but the nasal septum deviation was more common among the subjects with retruded and normally positioned maxilla as compared to the subjects with protruded maxilla. Thus, nasal septum deviation could be the reason for inadequate maxillary growth in the sagittal plane among subjects with retruded maxilla. To our knowledge, there is not even a single study to support our findings. But in contrast to our finding among subjects of groups I, II and III, group IV subjects in whom nasal septum was deviated, there was almost equal distribution of sagittal maxillary growth dysplasias, and without any positive association between nasal septum deviation and growth discrepancy of maxilla in the sagittal plane. In the present study with small sample size, it was difficult to make a definite conclusion whether the deviation of nasal septum could affect the growth of maxilla in sagittal plane. Thus, further study should be carried out with large sample size to find out any association between discrepancy of maxillary growth and nasal septum deviation.

\section{CONCLUSION}

The following conclusion was drawn from the present study: There was no positive association between the deviation of nasal septum and the discrepancy of maxillary growth in the sagittal plane. However, nasal septum deviation was more common among subjects of normal and retruded maxilla than among subjects with protruded maxilla.

\section{REFERENCES}

1. Scott JH. The cartilage of nasal septum: A contribution to the study of facial growth. Br Dent J 1953;95:37-44.

2. Scott JH. The analysis of facial growth from fetal life to adulthood. Angle Orthod 1963;33:110-13.

3. Babula WJ, Smiley GR, Dixon AD. The role of the cartilaginous nasal septum in midfacial growth. Am J Orthod 1970;58: 251-63.

4. Copray JC. Growth of the nasal septal cartilage of the rat in vitro. J Anat 1986;144:99-111.

5. Latham RA. The septo-premaxillary ligament and maxillary development. J Anat 1969;104:584-86.

6. Enlow DH. Facial growth (3rd ed). Philadelphia: WB Saunders Company 1990.

7. Moss ML, Bromberg BE, Song IC, Eisenman G. The passive role of the nasal septal cartilage in midfacial growth. Plast Reconstr Surg 1968;41:536-42.

8. Moss ML, Salentijn L. The primary role of the functional matrices in facial growth. Am J Orthod 1969;55:566-77.

9. Grymer LF, Pallisgaard C, Melsen B. The nasal septum in relation to the development of the nasomaxillary complex: A study in identical twins. Laryngoscope 1991;101:863-68. 
10. Grymer LF, Bosch C. The nasal septum and the development of the midface. A longitudinal study of a pair of monozygotic twins. Rhinology 1997;35:6-10.

11. Pirsig W. Open question in nasal surgery in children. Rhinology 1986;24:37-40.

12. Cottle MF. The maxilla-premaxilla approach to extensive nasal septum surgery. Arch Otolaryngol 1958;68:301-07.

13. Dahlberg G. Statistical methods for medical and biological students. London: George Allen and Unwin Ltd 1940;122-32.

14. Takahashi R. The formation of the nasal septum and the etiology of septal deformity. The concept of evolutionary paradox. Acta Otolaryngol 1987;443 (Suppl):1-160.

15. Holt GR. Biomechanics of nasal septal trauma. Otolaryngol Clin North Am 1999;32:615-19.

16. Moss ML. The role of the nasal septal cartilage in midfacial growth. In factors affecting the growth of the midface (ed JA McNamara), monograph 6, Craniofacial Growth Series, University of Michigan, Ann Arbor 1976.

17. Graber TM. A study of the craniofacial growth and development in the cleft palate child from birth to six years of age. Early treatment of cleft lip and palate. International Symposium, Zirich, (ed Huber) Bern 1964.

18. Nakamura S, Savara BS, Thomas DR. Facial growth of children with cleft lip and/or palate. Cleft palate J 1972;9:119-31.

19. Bergland $\mathrm{O}$, Borchgrevink $\mathrm{H}$. The role of the nasal septum in man elucidated by the maxillary development in certain types of facial clefts. Scand J Plast Reconstr Surg 1974;8:42-48.

20. Pirsig W. Growth of the deviated septum and its influence on midfacial development. Facial Plast Surg 1992;8:224-32.

\section{ABOUT THE AUTHORS}

\section{Ajay Bansal}

Ex-Postgraduate Student, Unit of Orthodontics, Oral Health Sciences Centre, Postgraduate Institute of Medical Education and Research Chandigarh, India

\section{Ashok Kumar Utreja}

Professor and Head, Unit of Orthodontics, Oral Health Sciences Centre, Postgraduate Institute of Medical Education and Research Chandigarh, India

\section{Satinder Pal Singh (Corresponding Author)}

Additional Professor, Unit of Orthodontics, Oral Health Sciences Centre, Postgraduate Institute of Medical Education and Research Sector-12, Chandigarh-160012, India, Phone: +91-9914209840 e-mail: drspsingh_chd@yahoo.com

\section{Naresh Kumar Panda}

Professor and Head, Department of Otolaryngology, Postgraduate Institute of Medical Education and Research, Chandigarh, India

\section{Ashok Kumar Jena}

Assistant Professor, Unit of Orthodontics, Oral Health Sciences Centre Postgraduate Institute of Medical Education and Research Chandigarh, India 